\title{
TPC Welcome
}

\section{Welcome Message from the Technical Program Chairs}

Welcome to the Fourteenth IEEE International Conference on Pervasive Computing and Communications (PerCom 2016)! The PerCom conference has been held in varying locations throughout the world, and the 2016 PerCom conference will be held for the first time in Sydney, Australia. IEEE PerCom is established as the premier annual scholarly venue in pervasive computing and communications. The conference provides an opportunity to present original research results, to relay practical development experiences, and to spark ideas for new research directions.

The PerCom conference series represents a true international forum. During its fourteen-year history, the conference has been held in six countries around the world. This year's conference reflects the global community: our organizing and program committee members represent 20 countries and authors submitted papers from 34 different countries.

This year's conference was competitive. A total of 168 papers were submitted for review. Each paper was reviewed by at least three technical program committee (TPC) members and reviewers contributed additional discussion together with the technical program chair and vice-chairs to reach a consensus about the papers. Finally, a TPC meeting was organized in Arlington, Texas to discuss the results of the reviewing process and select the final papers for the technical program. TPC members were selected to assign a subset of papers to ensure the highest quality in the final papers. This year, 20 full-length papers and 5 concise papers were accepted for presentation, representing an acceptance rate of $15 \%$. These numbers support the ongoing and growing interest in pervasive computing and position PerCom as the the leading international conference from researchers working in this field.

The PerCom technical program includes seven technical paper presentation sessions, two keynote talks, and a panel discussion. The technical presentation sessions are centered on the themes of Sensor Data Mining, Energy Analytics, Activity Sensing, Mobile Assistance, Pervasive Health, Pervasive Transportation, Sensing and Applications, and Security. There are three candidates for the Mark Weiser Best Paper Award, which will be presented in a dedicated session. A set of workshops, a Demonstration session, a Work In Progress session, and a PhD forum complete the program.

Creating the PerCom 2016 program required the time and expertise of numerous contributors. We are grateful for the tremendous help of the 100 members of the technical program committee.

Lastly, we thank the PerCom community for their support of this premier conference. We hope you enjoy the PerCom 2016 conference and that you are inspired by the ideas found in these papers.

Christian Becker, Frank Dürr, Jamie Payton, Daniele Riboni 